\title{
DEEP MORPHOLOGICAL AND SPECTRAL STUDY OF THE SNR RCW 86 WITH FERMI-LAT
}

M. Ajello ${ }^{1}$, L. Baldini ${ }^{2,3}$, G. Barbiellini ${ }^{4,5}$, D. Bastieri ${ }^{6,7}$, R. Bellazzini ${ }^{8}$, E. Bissaldi ${ }^{9}$, E. D. Bloom ${ }^{3}$, R. Bonino ${ }^{10,11}$, E. Bottacini ${ }^{3}$, T. J. Brandt ${ }^{12}$, J. Bregeon ${ }^{13}$, P. Bruel ${ }^{14}$, R. Buehler ${ }^{15}$, G. A. Caliandro ${ }^{3,16}$, R. A. Cameron ${ }^{3}$, M. Caragiulo ${ }^{9,17}$, E. Cavazzuti ${ }^{18}$, E. Charles ${ }^{3}$, A. Chekhtman ${ }^{19}$, S. Ciprini ${ }^{18,20}$, J. Cohen-Tanugi ${ }^{13}$, B. Condon ${ }^{21}$, F. Costanza ${ }^{9}$, S. Cutini ${ }^{18,20,22}$, F. D’Ammando ${ }^{23,24}$, F. De Palma ${ }^{9,25}$, R. Desiante ${ }^{10,26}$, N. Di Lalla ${ }^{8}$, M. Di Mauro ${ }^{3}$, L. Di Venere ${ }^{9,17}$, P. S. Drell ${ }^{3}$, G. Dubner ${ }^{27}$, D. Dumora ${ }^{21}$, L. Duvidovich ${ }^{27}$, C. Favuzzi ${ }^{9,17}$, W. B. Focke ${ }^{3}$, P. Fusco, ${ }^{9}, 17$, F. Gargano ${ }^{9}$, D. Gasparrini ${ }^{18,20}$, E. Giacani ${ }^{27}$, N. Giglietto ${ }^{9,17}$, T. Glanzman ${ }^{3}$, D. A. Green ${ }^{28}$, I. A. Grenier ${ }^{29}$,

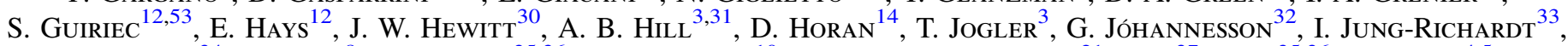

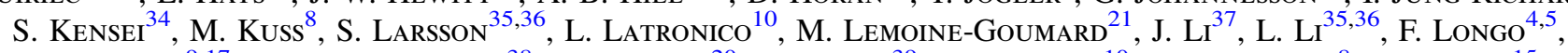
F. Loparco 9,17 , M. N. Lovellette ${ }^{38}$, P. Lubrano ${ }^{20}$, J. Magill ${ }^{39}$, S. Maldera ${ }^{10}$, A. Manfrede ${ }^{8}$, M. Mayer ${ }^{15}$,

M. N. Mazziotta ${ }^{9}$, J. E. McEnery ${ }^{12,39}$, P. F. Michelson ${ }^{3}$, W. Mitthumsiri ${ }^{40}$, T. Mizuno ${ }^{41}$, M. E. Monzani ${ }^{3}$, A. Morselli ${ }^{42}$, I. V. Moskalenko ${ }^{3}$, M. Negro ${ }^{10,11}$, E. Nuss ${ }^{13}$, M. Orientio ${ }^{23}$, E. Orlando ${ }^{3}$, J. F. Ormes ${ }^{43}$, D. Paneque ${ }^{3,44}$, J. S. Perkins ${ }^{12}$,

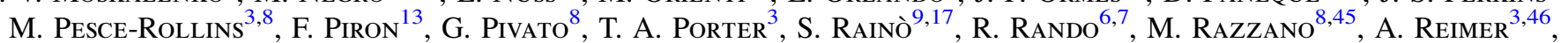

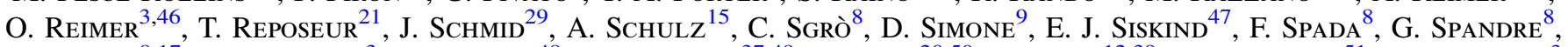

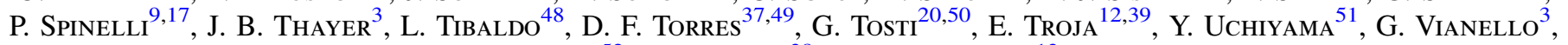
J. VINK ${ }^{52}$, K. S. WOOD ${ }^{38}$, AND M. YASSINE ${ }^{13}$

${ }^{1}$ Department of Physics and Astronomy, Clemson University, Kinard Lab of Physics, Clemson, SC 29634-0978, USA

${ }^{2}$ Università di Pisa and Istituto Nazionale di Fisica Nucleare, Sezione di Pisa, I-56127 Pisa, Italy

${ }^{3}$ W. W. Hansen Experimental Physics Laboratory, Kavli Institute for Particle Astrophysics and Cosmology, Department of Physics and SLAC National Accelerator Laboratory, Stanford University, Stanford, CA 94305, USA

${ }^{4}$ Istituto Nazionale di Fisica Nucleare, Sezione di Trieste, I-34127 Trieste, Italy

${ }^{5}$ Dipartimento di Fisica, Università di Trieste, I-34127 Trieste, Italy

${ }^{6}$ Istituto Nazionale di Fisica Nucleare, Sezione di Padova, I-35131 Padova, Italy

${ }^{7}$ Dipartimento di Fisica e Astronomia "G. Galilei," Università di Padova, I-35131 Padova, Italy

${ }^{8}$ Istituto Nazionale di Fisica Nucleare, Sezione di Pisa, I-56127 Pisa, Italy

${ }^{9}$ Istituto Nazionale di Fisica Nucleare, Sezione di Bari, I-70126 Bari, Italy; micaela.caragiulo@ba.infn.it

${ }^{10}$ Istituto Nazionale di Fisica Nucleare, Sezione di Torino, I-10125 Torino, Italy

${ }^{11}$ Dipartimento di Fisica Generale "Amadeo Avogadro," Università degli Studi di Torino, I-10125 Torino, Italy

${ }^{12}$ NASA Goddard Space Flight Center, Greenbelt, MD 20771, USA

${ }^{13}$ Laboratoire Univers et Particules de Montpellier, Université Montpellier, CNRS/IN2P3, Montpellier, France

${ }^{14}$ Laboratoire Leprince-Ringuet, École polytechnique, CNRS/IN2P3, Palaiseau, France

${ }^{15}$ Deutsches Elektronen Synchrotron DESY, D-15738 Zeuthen, Germany

${ }^{16}$ Consorzio Interuniversitario per la Fisica Spaziale (CIFS), I-10133 Torino, Italy

${ }^{17}$ Dipartimento di Fisica "M. Merlin" dell’Università e del Politecnico di Bari, I-70126 Bari, Italy ${ }^{18}$ Agenzia Spaziale Italiana (ASI) Science Data Center, I-00133 Roma, Italy

${ }^{19}$ College of Science, George Mason University, Fairfax, VA 22030, resident at Naval Research Laboratory, Washington, DC 20375, USA

${ }^{20}$ Istituto Nazionale di Fisica Nucleare, Sezione di Perugia, I-06123 Perugia, Italy

${ }^{21}$ Centre d'Études Nucléaires de Bordeaux Gradignan, IN2P3/CNRS, Université Bordeaux 1, BP120, F-33175 Gradignan Cedex, France; condon@cenbg.in2p3.fr, lemoine@cenbg.in2p3.fr

22 INAF Osservatorio Astronomico di Roma, I-00040 Monte Porzio Catone (Roma), Italy

${ }^{23}$ INAF Istituto di Radioastronomia, I-40129 Bologna, Italy

${ }^{24}$ Dipartimento di Astronomia, Università di Bologna, I-40127 Bologna, Italy

${ }^{25}$ Università Telematica Pegaso, Piazza Trieste e Trento, 48, I-80132 Napoli, Italy ${ }^{26}$ Università di Udine, I-33100 Udine, Italy

${ }^{27}$ Instituto de Astronomía y Fisica del Espacio, Parbellón IAFE, Cdad. Universitaria, Buenos Aires, Argentina ${ }^{28}$ Cavendish Laboratory, Cambridge CB3 OHE, UK

${ }^{29}$ Laboratoire AIM, CEA-IRFU/CNRS/Université Paris Diderot, Service d'Astrophysique, CEA Saclay, F-91191 Gif sur Yvette, France

${ }^{30}$ University of North Florida, Department of Physics, 1 UNF Drive, Jacksonville, FL 32224, USA

${ }^{31}$ School of Physics and Astronomy, University of Southampton, Highfield, Southampton, SO17 1BJ, UK

${ }^{32}$ Science Institute, University of Iceland, IS-107 Reykjavik, Iceland

${ }^{33}$ Friedrich-Alexander-Universität, Erlangen-Nürnberg, Schlossplatz 4, D-91054 Erlangen, Germany

${ }_{35}^{34}$ Department of Physical Sciences, Hiroshima University, Higashi-Hiroshima, Hiroshima 739-8526, Japan

35 Department of Physics, KTH Royal Institute of Technology, AlbaNova, SE-106 91 Stockholm, Sweden

The Oskar Klein Centre for Cosmoparticle Physics, AlbaNova, SE-106 91 Stockholm, Sweden

${ }^{37}$ Institute of Space Sciences (IEEC-CSIC), Campus UAB, E-08193 Barcelona, Spain

${ }^{38}$ Space Science Division, Naval Research Laboratory, Washington, DC 20375-5352, USA

${ }^{39}$ Department of Physics and Department of Astronomy, University of Maryland, College Park, MD 20742, USA

${ }^{40}$ Department of Physics, Faculty of Science, Mahidol University, Bangkok 10400, Thailand

${ }^{41}$ Hiroshima Astrophysical Science Center, Hiroshima University, Higashi-Hiroshima, Hiroshima 739-8526, Japan

${ }^{42}$ Istituto Nazionale di Fisica Nucleare, Sezione di Roma "Tor Vergata," I-00133 Roma, Italy

${ }^{43}$ Department of Physics and Astronomy, University of Denver, Denver, CO 80208, USA ${ }^{44}$ Max-Planck-Institut für Physik, D-80805 München, Germany

${ }^{45}$ Funded by contract FIRB-2012-RBFR12PM1F from the Italian Ministry of Education, University and Research (MIUR)

${ }^{46}$ Institut für Astro- und Teilchenphysik and Institut für Theoretische Physik, Leopold-Franzens-Universität Innsbruck, A-6020 Innsbruck, Austria

NYCB Real-Time Computing Inc., Lattingtown, NY 11560-1025, USA

${ }^{48}$ Max-Planck-Institut für Kernphysik, D-69029 Heidelberg, Germany

${ }^{49}$ Institució Catalana de Recerca i Estudis Avançats (ICREA), Barcelona, Spain

${ }^{50}$ Dipartimento di Fisica, Università degli Studi di Perugia, I-06123 Perugia, Italy 


\author{
${ }^{51}$ Department of Physics, 3-34-1 Nishi-Ikebukuro, Toshima-ku, Tokyo 171-8501, Japan \\ 52 Anton Pannekoek Institute for Astronomy, University of Amsterdam, Science Park 904, 1098 XH Amsterdam, The Netherlands \\ Received 2015 December 14; accepted 2016 January 24; published 2016 March 2
}

\section{ABSTRACT}

RCW 86 is a young supernova remnant (SNR) showing a shell-type structure at several wavelengths and is thought to be an efficient cosmic-ray (CR) accelerator. Earlier Fermi Large Area Telescope results reported the detection of $\gamma$-ray emission coincident with the position of RCW 86 but its origin (leptonic or hadronic) remained unclear due to the poor statistics. Thanks to 6.5 years of data acquired by the Fermi-LAT and the new event reconstruction Pass 8 , we report the significant detection of spatially extended emission coming from RCW 86. The spectrum is described by a power-law function with a very hard photon index $\left(\Gamma=1.42 \pm 0.1_{\text {stat }} \pm 0.06_{\text {syst }}\right)$ in the $0.1-500 \mathrm{GeV}$ range and an energy flux above $100 \mathrm{MeV}$ of $\left(2.91 \pm 0.8_{\text {stat }} \pm 0.12_{\text {syst }}\right) \times 10^{-11} \mathrm{erg} \mathrm{cm}^{-2} \mathrm{~s}^{-1}$. Gathering all the available multiwavelength (MWL) data, we perform a broadband modeling of the nonthermal emission of RCW 86 to constrain parameters of the nearby medium and bring new hints about the origin of the $\gamma$ ray emission. For the whole SNR, the modeling favors a leptonic scenario in the framework of a two-zone model with an average magnetic field of $10.2 \pm 0.7 \mu \mathrm{G}$ and a limit on the maximum energy injected into protons of $2 \times 10^{49} \mathrm{erg}$ for a density of $1 \mathrm{~cm}^{-3}$. In addition, parameter values are derived for the north-east and south-west (SW) regions of RCW 86, providing the first indication of a higher magnetic field in the SW region.

Key words: acceleration of particles - cosmic rays - ISM: individual objects (RCW 86)

\section{INTRODUCTION}

It is widely believed that supernova remnants (SNRs) are the primary sources of Galactic cosmic rays (CRs) observed on Earth, up to the knee energy at $\sim 10^{15} \mathrm{eV}$, as first proposed by Ginzburg \& Syrovatsky (1961). CRs can be accelerated to very high energies at collisionless shocks driven by Supernova (SN) explosions through diffusive shock acceleration (DSA; Axford et al. 1977; Krymskii 1977; Bell 1978a, 1978b; Blandford \& Ostriker 1978). During this process the kinetic energy released in SN explosions has to be transferred to CRs with an efficiency of $\sim 10 \%$ (Ginzburg \& Syrovatskii 1964). A description of the acceleration process can be achieved within a nonlinear DSA theory (Malkov \& Drury 2001) with magnetic field amplification, probably by accelerated particles themselves, due to streaming instability (Bell 2004; Amato \& Blasi 2006; Caprioli et al. 2008). The strong evidence for large magnetic fields in the shock region is given by the observation of narrow filaments of non-thermal X-ray radiation in young SNRs (Ballet 2006; Vink 2012). The non-thermal emission produced through the interaction of accelerated particles with radiation and/or matter in the environment of the SNR, via synchrotron (SC), inverse Compton (IC), non-thermal bremsstrahlung, hadronic interactions and subsequent $\pi^{0}$ decay, gives information about the particle acceleration mechanisms at work in these sources.

RCW 86 (Rodgers et al. 1960), also known as MSH 14-63 (Mills et al. 1961) or G315.4-2.3, is a SNR located in the southern sky. The origin of this SNR is still debated but recent studies (Williams et al. 2011; Broersen et al. 2014) suggest that RCW 86 is associated to the historical SN 185 (Stephenson \& Green 2002) and is the result of a Type Ia explosion, also supported by the large amount of $\mathrm{Fe}\left(\sim 1 M_{\odot}\right.$; Yamaguchi et al. 2011). A large shell ( $\sim 40^{\prime}$ in diameter $)$ is clearly detected in radio (Kesteven \& Caswell 1987), optical (Smith 1997), infrared (Williams et al. 2011), X-rays (Pisarski et al. 1984) and very-high-energy (VHE; $E>0.1 \mathrm{TeV}$ ) $\gamma$-rays (Aharonian et al. 2009; H. E. S. S. Collaboration et al. 2016). At highenergy (HE, $0.1<E<100 \mathrm{GeV}$ ) $\gamma$-rays, Lemoine-Goumard et al. (2012) derived upper limits on the flux and Yuan et al.

\footnotetext{
$\overline{53}$ NASA Postdoctoral Program Fellow, USA.
}

(2014) reported the detection of a pointlike $\gamma$-ray source matching the position of RCW 86. In 2015, RCW 86 was reported for the first time as an extended source (with a radius of 0.27 above $50 \mathrm{GeV}$ ) in the Second Catalog of Hard FermiLAT Sources (The Fermi-LAT Collaboration 2015).

X-ray observations of RCW 86 reveal a non-spherically symmetric shell with both thermal $(0.5-2 \mathrm{keV})$ and nonthermal $(2-5 \mathrm{keV})$ emission, with different morphologies. The soft X-rays are related to optical emission from non-radiative shocks and IR emission from collisionally heated dust, whereas the hard X-ray continuum, located mostly in the southwestern part of the remnant, is due to $\mathrm{SC}$ radiation coming from electrons accelerated at the reverse shock of the remnant, as suggested by its spatial correlation with the strong $\mathrm{Fe}-\mathrm{K}$ line emission (Rho et al. 2002). Using Suzaku telescope data, Ueno et al. (2007) produced a map of the $\mathrm{Fe}-\mathrm{K}$ line emission in the southwestern part of the remnant, showing that the $\mathrm{Fe}-\mathrm{K}$ line emission correlates well with the radio SC emission. Furthermore, the higher temperature plasma, which mostly contains the strong $\mathrm{Fe}-\mathrm{K}$ line emission, suggests that this line originates from Fe-rich ejecta heated by a reverse shock (Ueno et al. 2007; Yamaguchi et al. 2008). This X-ray SC radiation is produced by $\mathrm{TeV}$ electrons accelerated at the shock as confirmed by the VHE $\gamma$-ray emission detected with the H.E.S. S. experiment (Aharonian et al. 2009; H. E. S. S. Collaboration et al. 2016).

The distance of RCW 86 is estimated to be $2.5 \pm 0.5 \mathrm{kpc}$ through the recent proper motion measurements by Helder et al. (2013), combined with plasma temperature measurements based on the broad $\mathrm{H} \alpha$ lines. The ambient density around RCW 86 is inhomogeneous and the shock speed value, as well as the magnetic field, change along the shell-like structure. In particular, in the southwest and northwest regions shocks are slow, around $\sim 600-800 \mathrm{~km} \mathrm{~s}^{-1}$ (Long \& Blair 1990; Ghavamian et al. 2001), and post-shock densities are relatively high $\left(\sim 2 \mathrm{~cm}^{-3}\right.$; Williams et al. 2011). Whereas, faster shocks $\left(\sim 2700 \mathrm{~km} \mathrm{~s}^{-1}\right.$ and $6000 \pm 2800 \mathrm{~km} \mathrm{~s}^{-1}$; Vink et al. 2006; Helder et al. 2009) and lower densities ( 0.1-0.3 $\mathrm{cm}^{-3}$; Yamaguchi et al. 2008) have been measured in the north-east (NE) region. The large size of this young remnant as well as the asymmetry in its morphology can be explained by an off-center 
explosion in a low-density cavity, as proposed by Williams et al. (2011).

Here we present the results of a deep morphological analysis with the new Fermi-LAT event reconstruction set, Pass 8, as well as a study of the broadband emission using the available information in the radio, X-ray and VHE $\gamma$-ray domains.

\section{Fermi-LAT AND PASS 8 DESCRIPTION}

The Fermi-LAT is a $\gamma$-ray telescope which detects photons by conversion into electron-positron pairs in the energy range between $20 \mathrm{MeV}$ to higher than $500 \mathrm{GeV}$, as described in Atwood et al. (2009). The LAT is made of a high-resolution converter/tracker (for direction measurement of the incident $\gamma$ rays), a CsI(Tl) crystal calorimeter (for energy measurement), and an anti-coincidence detector to identify the background by charged particles. The LAT has a large effective area $\left(\sim 8200 \mathrm{~cm}^{2}\right.$ on-axis above $\left.1 \mathrm{GeV}\right)$, a wide field of view $(\sim 2.4 \mathrm{sr})$ as well as good angular resolution (with a $68 \%$ containment radius of $\sim 0^{\circ} .8$ at $1 \mathrm{GeV}$ ).

Since the launch of the spacecraft in 2008 June, the LAT event-level analysis has been periodically upgraded to take advantage of the increasing knowledge of the Fermi-LAT functioning as well as the environment in which it operates. Following Pass 7, released in 2011 August, Pass 8 is the latest version of the Fermi-LAT data. ${ }^{54}$ The development of Pass 8 was the result of a long-term effort aimed at a radical revision of the entire event-level analysis and tends to realize the full scientific potential of the LAT (Atwood et al. 2013). Combining the improvement of the effective area, the point-spread function (PSF) and the energy resolution with the large amount of data collected by the LAT since its launch, Pass 8 is a powerful tool to identify and study extended $\gamma$-ray sources.

\section{Fermi-LAT OBSERVATIONS AND DATA ANALYSIS}

We analyzed 6.5 years of data collected between 2008 August 4th and 2015 January 31 stwithin a $15^{\circ} \times 15^{\circ}$ region centered on the position of RCW 86. We used events with energies between $100 \mathrm{MeV}$ and $500 \mathrm{GeV}$ with a maximum zenith angle of $100^{\circ}$ to limit the contamination due to the Earth Limb. To assure good quality events, we excluded the time intervals when the Fermi spacecraft was within crossed the South Atlantic Anomaly were excluded. We used the version $+10-00-03+$ of the ScienceTools and the +P8R2_V6+ Instrument Response Functions (IRFs) with the event class +SOURCE+, which corresponds to the best compromise between the number of selected photons and the charged particle residual background for the study of point-like or slightly extended sources.

Two tools were used for the analysis: $+g t l i k e+$ for the spectral analysis and tpointlike+ for the spatial analysis. +gtlike+ is a binned maximum likelihood method (Mattox et al. 1996) implemented in the Fermi Science Tools. tpointlike+ is an alternative code used for fast analysis of Fermi-LAT data and able to characterize the extension of a source (Kerr 2011). These tools fit a source model to the data along with models for the residual charged particles and diffuse $\gamma$-ray emission. The Galactic diffuse emission was modeled by the standard LAT diffuse emission ring-hybrid model $+g l l_{-}$iem_v06.fits+ and the residual background and

\footnotetext{
${ }_{54}$ Passes correspond to the release of upgraded versions of the LAT eventlevel analysis framework.
}

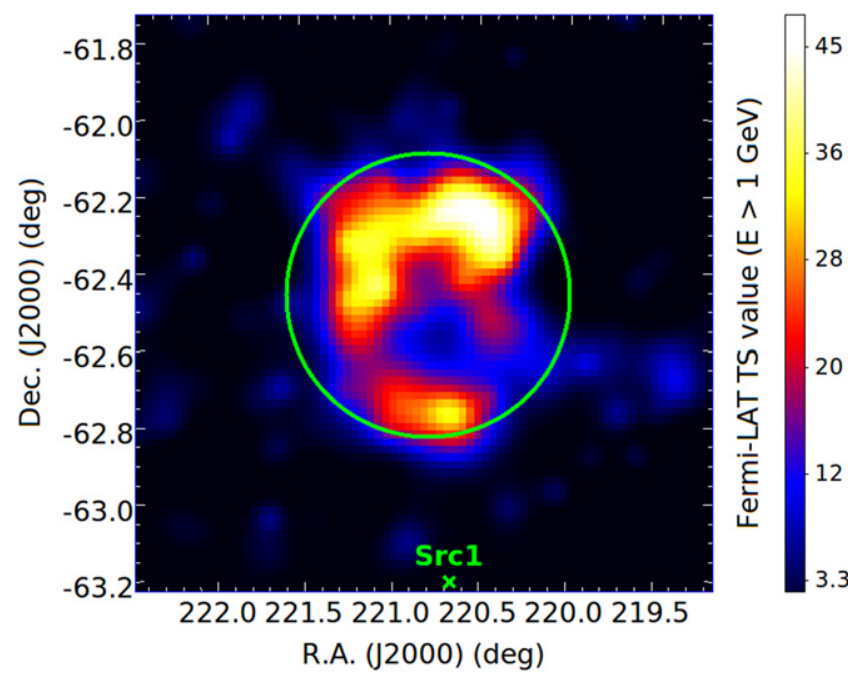

Figure 1. Test Statistic (TS) map above $1 \mathrm{GeV}$ centered on RCW 86. The green cross indicates a point-like source that has been added to the source model to fit background emissions in the vinicity of RCW 86 .

extragalactic radiation were described by a single isotropic component with the spectral shape in the tabulated model +iso_P8R2_SOURCE_V6_V06.txt+. The models are available from the Fermi Science Support Center. Sources located in the $15^{\circ} \times 15^{\circ}$ region centered on RCW 86 and included in the Fermi-LAT Third Source Catalog (Acero et al. 2015a, hereafter 3FGL), based on the first four years of Pass 7 data, were added to our spectral-spatial model of the region. Only sources within $5^{\circ}$ around the position of RCW 86 were refitted, in addition to the Galactic diffuse and isotropic emission. The energy dispersion, which is defined in terms of the fractional difference between the reconstructed energy and the true energy of the events, was taken into account in both spatial and spectral analysis to consider the imperfection of the energy reconstruction.

\subsection{Morphological Analysis}

As it is critical to have a better angular resolution for the morphological analysis, we perform the spatial analysis above $1 \mathrm{GeV}$. The resulting PSF is $\sim 0.27$ for a photon index of 1.5 (see Section 3.2). We first fitted the $15^{\circ} \times 15^{\circ}$ region centered on RCW 86 with the 3FGL sources, the Galactic diffuse and isotropic emission templates and computed the Fermi-LAT Test Statistic (TS) map $\left(4^{\circ} \times 4^{\circ}\right.$ with $0.02 \mathrm{pixel}^{-1}$, see Figure 1). The $T S$ is defined as twice the difference between the $\log$-likelihood $L_{1}$ obtained by fitting a source model plus the background model to the data, and the $\log$-likelihood $L_{0}$ obtained by fitting the background model only, i.e $T S=2\left(L_{1}-L_{0}\right)$. Figure 1 contains the $T S$ value for a point source of fixed photon index $\Gamma=2$ in each pixel of the map, thus giving a measure of the statistical significance for the detection of a $\gamma$-ray source with that spectrum in excess of the background. The TS map revealed a significant emission coincident with the position of RCW 86, coming essentially from the NE region of the remnant (there is no significant variation of the background emission within 0.5 ). Additional excess $\gamma$-ray emissions were also detected in the region of interest. Therefore, we added four new sources, denoted with the identifiers $\mathrm{Src} 1, \mathrm{Src} 2, \mathrm{Src} 3$, and $\mathrm{Src} 4$ (only Src1 is visible in the field of view of Figure 1), in our model to take into account 
Table 1

Centroid and Extension Fits of the LAT Data using Pointlike above $1 \mathrm{GeV}$

\begin{tabular}{|c|c|c|c|c|c|c|}
\hline Spatial Model & $T S_{\text {ext }}$ & R.A. ( $\left.{ }^{\circ}\right)$ & Decl. $\left({ }^{\circ}\right)$ & Radius $\left(^{\circ}\right)$ & Inner Radius $\left({ }^{\circ}\right)$ & $N_{\text {dof }}$ \\
\hline Point Source & $\cdots$ & $220.56 \pm 0.07$ & $-62.25 \pm 0.02$ & $\cdots$ & $\cdots$ & 3 \\
\hline Disk & 68 & $220.73 \pm 0.04$ & $-62.47 \pm 0.03$ & $0.37 \pm 0.02$ & $\cdots$ & 4 \\
\hline Ring & 75 & $220.74 \pm 0.02$ & $-62.51 \pm 0.02$ & $0.31 \pm 0.02$ & $0.21 \pm 0.02$ & 5 \\
\hline
\end{tabular}

Note. Uncertainties are statistical errors ( $68 \%$ containment). $N_{\text {dof }}$ corresponds to the number of degrees of freedom for each model.

these signals. These regions of $\gamma$-ray emission are considered to belong to background sources not detected in 3FGL due to the larger dataset and the increased effective area of Pass 8 data.

To determine the best morphology of RCW 86, the data were fitted with different spatial models (point-source, disk, ring) while fitting the spectrum of the source (normalization and spectral index) simultaneously. The results of this first analysis are reported in Table 1. The significance of the extension of RCW 86 is quantified with $T S_{\text {ext }}$, which is defined as twice the difference between the log-likelihood of an extended source model and the log-likelihood of a point-like source model. For the uniform disk hypothesis, the source is significantly extended $\left(T S_{\text {ext }}=68\right.$, which corresponds to a significance of $\sim 8 \sigma$ for the extension) with respect to the LAT PSF. The fitted radius, $0.37 \pm 0.02_{\text {stat }}$, is in good agreement with the size of the SNR as seen in radio (Kesteven \& Caswell 1987), infrared (Williams et al. 2011), X-rays (Pisarski et al. 1984) and VHE $\gamma$ rays (H. E. S. S. Collaboration et al. 2016). Using a ring as a spatial model, the log-likelihood is improved in comparison to that obtained with a uniform disk by only $2.6 \sigma$, which is not enough to claim that the source has a shell-like morphology. The spatial analysis was confirmed by tgtlike+, as shown in Table 2.

In addition to geometrical models, we have fitted the LAT data with MWL morphological templates to evaluate the correspondance of the $\gamma$-ray emission above $1 \mathrm{GeV}$ coming from RCW 86 with different source morphologies (see Figure 2). For that purpose, we compared the $T S$ obtained with the best-fit uniform disk model (see Table 1) and the ones obtained with the MWL morphological templates. The radio data are from the Molonglo Observatory Synthesis Telescope (MOST) at $843 \mathrm{MHz}$ (Murphy et al. 2007) and the TeV data from H.E.S.S. (H. E. S. S. Collaboration et al. 2016). Concerning the X-ray data, we used the observations of the space telescope XMM-Newton, in the energy range 0.5-1 and 2-5 keV (Broersen et al. 2014), to estimate the correlation with thermal and non-thermal X-ray emission separately. The analysis revealed a good match between the $\mathrm{HE} \gamma$-ray emission and the VHE $\gamma$-ray and non-thermal X-ray emissions. However, the radio and thermal X-ray signals do not fit well with the HE $\gamma$-ray emission.

As RCW 86 is known to present an asymmetry in its morphology from radio to VHE $\gamma$-rays (Broersen et al. 2014), we divided the spatial models and fit separately the two halftemplates in order to quantify the difference between the NE and the south-west (SW) region of the SNR, using the improved PSF of the Pass 8 data. We determined the best (most significant) angle of division by computing the log-likelihood for 18 regularly spaced angles, from $0^{\circ}$ to $170^{\circ}, 0^{\circ}$ corresponding to a division along the line north/south in equatorial coordinates (the white dashed line in Figure 3). This analysis was performed for all the templates and we obtained
Table 2

TS Values Obtained with Gtlike above $1 \mathrm{GeV}$

\begin{tabular}{lcc}
\hline \hline Template & $T S$ & $N_{\text {dof }}$ \\
\hline Point Source & 34 & 4 \\
Disk & $97(99)$ & $5(8)$ \\
Ring & $104(105)$ & $6(9)$ \\
MOST (843 MHz) & $77(86)$ & $2(5)$ \\
XMM-Newton $(0.5-1 \mathrm{keV})$ & $62(90)$ & $2(5)$ \\
XMM-Newton $(2-5 \mathrm{keV})$ & $91(101)$ & $2(5)$ \\
H.E.S.S. & $98(100)$ & $2(5)$ \\
\hline
\end{tabular}

Note. Numbers in parentheses correspond to the values obtained after fitting separately the two hemispheres shown in Figure 3.

the same best angle (the green dashed line in Figure 3) for all of them.

By comparing the results for non-divided and divided templates presented in Table 2, we notice that the division improves significantly the likelihood for the MOST and the $X M M-N e w t o n$ templates. Moreover, the non-thermal X-ray template is as good as the disk and the H.E.S.S. templates when it is divided. This indicates that the $\mathrm{X}$-ray and radio morphologies do not reproduce well the HE $\gamma$-ray signal in the case of a single region, as it will be confirmed in Section 7 with the broadband modeling of the spectrum. However, the likelihood is not much improved when dividing the disk and the H.E.S.S. template, showing that they well reproduce the whole SNR as seen with Fermi-LAT. The non-divided H.E.S. S. template provides the highest TS of all the non-divided (and divided when taking into account the number of degrees of freedom) templates when fitting the $\mathrm{HE} \gamma$-ray emission. As a consequence, this template was used to perform the spectral analysis.

\subsection{Spectral Analysis}

To study the spectrum of RCW 86, we performed a maximum likelihood fit with $+g t l i k e+$ in the energy range $100 \mathrm{MeV}-500 \mathrm{GeV}$, using the non-divided H.E.S.S. template as a spatial model. The Fermi-LAT data are well described by a power law function $(T S=99)$, with a photon index of $1.42 \pm 0.1_{\text {stat }} \pm 0.06_{\text {syst }}$ and an energy flux above $100 \mathrm{MeV}$ of $\left(2.91 \pm 0.8_{\text {stat }} \pm 0.12_{\text {syst }}\right) \times 10^{-11} \mathrm{erg} \mathrm{cm}^{-2} \mathrm{~s}^{-1}$. One should note that the photon index $\Gamma$ is linked to the radio energy index $\alpha$ (defined as $S_{\nu} \propto \nu^{-\alpha}$ ) by the relation $\Gamma=1+\alpha$. For RCW 86, $\alpha$ is found to be 0.6 and is therefore consistent with a hard index in $\mathrm{GeV}$. We also performed several fits while fixing the index of the power law at different values: 1.5, 1.6, 1.7 and 1.8. In each case, we measured the deterioration of the loglikelihood by computing the difference between the $T S$ of the best-fit and the $T S$ obtained with the fixed index. As a result, we excluded $\Gamma>1.7$ at more than $3 \sigma$. Although a broken power-law seems more likely when considering both Fermi- 

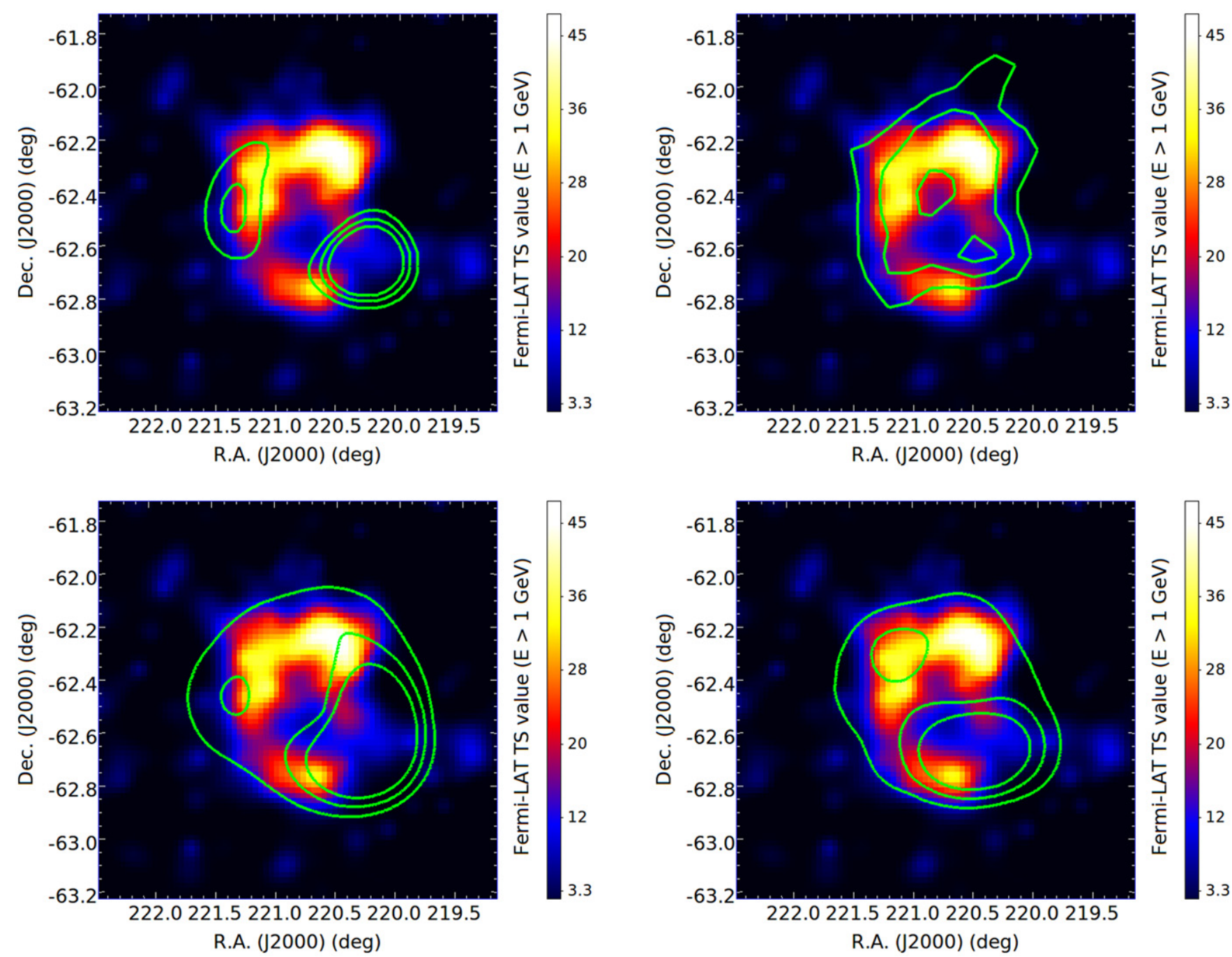

Figure 2. Test Statistic (TS) map above $1 \mathrm{GeV}$ centered on RCW 86 with MWL contours. All data sets have been smoothed such that their angular resolution is similar to the Fermi-LAT PSF ( 0.27 at $68 \%$ C.L.). The radio (top-left), TeV (top-right), thermal (bottom-left) and non-thermal (bottom-right) X-ray data are from Murphy et al. (2007), H. E. S. S. Collaboration et al. (2016) and Broersen et al. (2014) respectively.

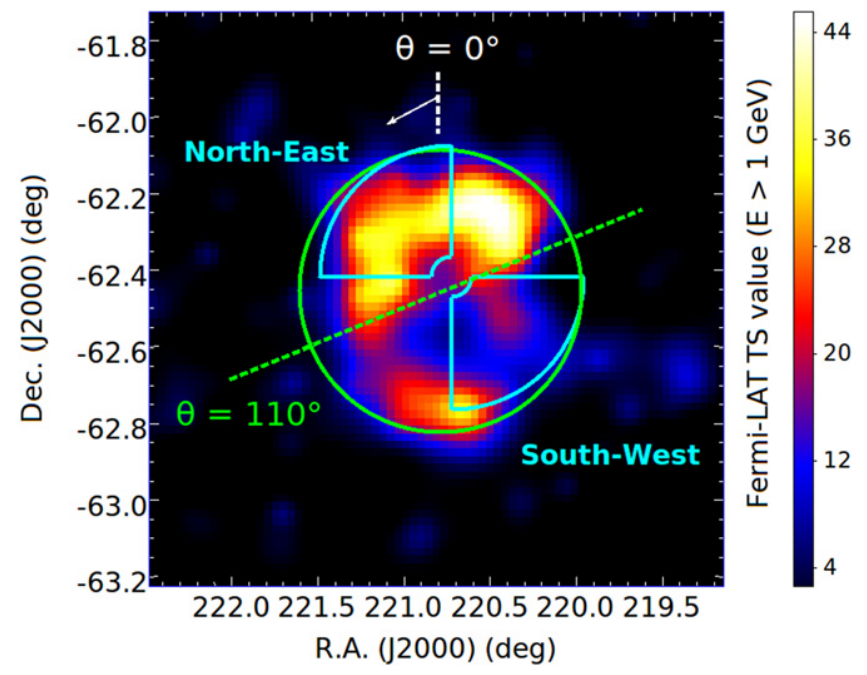

Figure 3. The white dashed line corresponds to the north/south line in equatorial coordinates $\left(\theta=0^{\circ}\right)$ while the green one represents the best angle of division $\left(\theta=110^{\circ}\right)$. The green circle corresponds to the best-fit disk model provided by pointlike in Table 1 and the cyan quadrants represent the two regions defined in H. E. S. S. Collaboration et al. (2016) that are studied in Section 3.2.
LAT and H.E.S.S. data, the log-likelihood is improved by only $2 \sigma(\Delta T S=7$ for 2 more degrees of freedom) when fitting the spectra with this function. The four additional background sources (Src1, Src2, Src3, and Src4) were taken into account in these fits and their best-fit positions and spectral parameters are given in Table 3. Despite having a TS lower than 25, Src1 and Src2 were kept in our background model to avoid any contamination to the RCW 86 spectrum.

Systematic errors are defined as $\operatorname{Err}_{\mathrm{syst}}=$ $\sqrt{\left(\operatorname{Err}_{\text {iem }}\right)^{2}+\left(\operatorname{Err}_{\text {irf }}\right)^{2}+\left(\operatorname{Err}_{\text {model }}\right)^{2}}$. This expression takes into account the imperfection of the Galactic diffuse emission model $\left(\operatorname{Err}_{i e m}\right)$, uncertainties in the effective area calibration $\left(\operatorname{Err}_{\text {irf }}\right)$ and uncertainties on the source shape $\left(\operatorname{Err}_{\text {model }}\right)$. The first one was estimated by using alternative Interstellar Emission Models (IEMs), as described in Acero et al. (2015b). For the second one, we applied scaling functions that change the effective area $( \pm 3 \%$ for $100 \mathrm{MeV}-100 \mathrm{GeV}$ and $\pm 3 \%+10 \% \times(\log (E / \mathrm{MeV})-5)$ above $100 \mathrm{GeV}$ Ackermann et al. 2012). The third one was obtained by fitting the $\gamma$-ray emission with the best spatial models (the disk and the ring) provided by pointlike+. 
Table 3

Best Spectral Fit Parameters Obtained for the Nearby Background Sources Src1, Src2, Src3 and Src4 with Gtlike using the H.E.S.S.

\begin{tabular}{|c|c|c|c|c|c|}
\hline Source Name & R.A. $\left({ }^{\circ}\right)$ & Decl. $\left({ }^{\circ}\right)$ & Spectral Index & Flux $\left(\times 10^{-12} \mathrm{erg}^{-1} \mathrm{~cm}^{-2} \mathrm{~s}^{-1}\right)$ & $\overline{T S}$ \\
\hline$\overline{S r c 1}$ & $220.66 \pm 0.05$ & $-63.21 \pm 0.02$ & $1.69 \pm 0.20$ & $5.11_{-2.51}^{+3.41}$ & 24 \\
\hline Src2 & $223.15 \pm 0.07$ & $-63.00 \pm 0.04$ & $1.82 \pm 0.24$ & $4.19_{-2.25}^{+3.41}$ & 18 \\
\hline Src3 & $224.15 \pm 0.14$ & $-63.22 \pm 0.05$ & $2.56 \pm 0.13$ & $7.11_{-1.65}^{+1.53}$ & 26 \\
\hline Src4 & $222.50 \pm 0.09$ & $-60.36 \pm 0.05$ & $2.54 \pm 0.12$ & $15.3_{-2.61}^{+2.67}$ & 52 \\
\hline
\end{tabular}

Note. Template for RCW 86 above $100 \mathrm{MeV}$. The errors are statistical errors only.

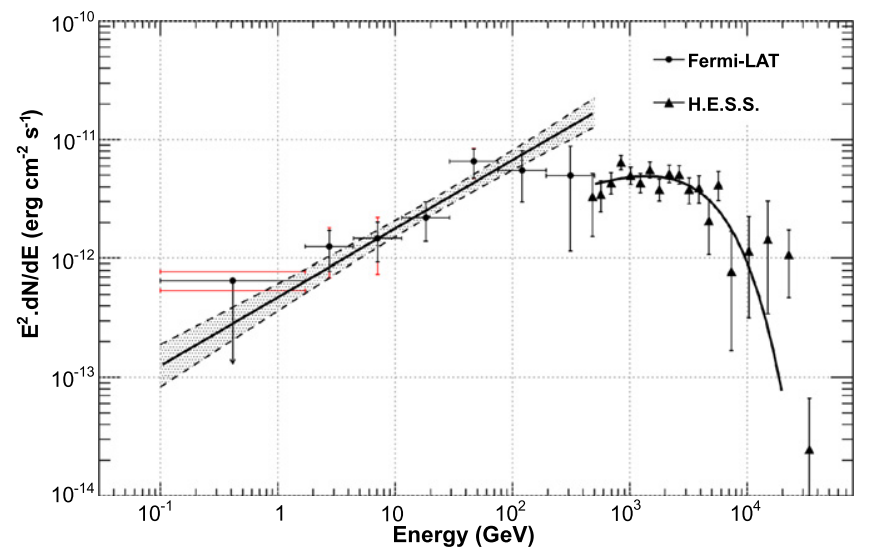

Figure 4. Spectral Energy Distribution of RCW 86 from $100 \mathrm{MeV}$ to $50 \mathrm{TeV}$ with the Fermi-LAT and H.E.S.S. (H. E. S. S. Collaboration et al. 2016) data points shown as black circles and triangles, respectively. The black solid line passing through the H.E.S.S. points corresponds to the best-fit spectrum from H. E. S. S. Collaboration et al. (2016). The smaller and larger errors on the Fermi fluxes are statistical and total errors (quadratic sum of statistical and systematic errors), respectively. The range of upper limit values (black and red lines) correspond to the uncertainty in the diffuse modeling. The dark gray shaded area (delimited by black dashed lines) represents the $68 \%$ confidence band of the fitted Fermi-LAT spectrum.

Figure 4 shows the Spectral Energy Distribution (SED) of RCW 86. The Fermi-LAT spectral points were obtained by dividing the $100 \mathrm{MeV}-500 \mathrm{GeV}$ range into nine logarithmically spaced energy bins. We assumed a power law with a spectral index of 1.4 and used the H.E.S.S. spatial model. In addition to RCW 86, only very bright and close sources $\left(T S>500\right.$ and within a radius of $\left.5^{\circ}\right)$, as well as the Galactic diffuse and the isotropic emission were fitted. We derived the $95 \%$ C.L. upper limit in the $0.1-1.7 \mathrm{GeV}$ band, combining the first three bins in which no signal was detected by the Fermi-LAT.

To pursue our purpose of understanding the variation of the physical conditions in RCW 86, we performed a spectral analysis between $100 \mathrm{MeV}$ and $500 \mathrm{GeV}$ using the H.E.S.S. template divided in half along the green dashed line shown in Figure 3. We obtained an index of $1.36 \pm 0.17_{\text {stat }}$ and an energy flux above $100 \mathrm{MeV}$ of $(1.69 \pm 0.63) \times$ $10^{-11} \mathrm{erg} \mathrm{cm}^{-2} \mathrm{~s}^{-1}$ for the upper region and an index of $1.62 \pm 0.17_{\text {stat }}$ and an energy flux of $(0.97 \pm 0.31) \times$ $10^{-11} \mathrm{erg} \mathrm{cm}^{-2} \mathrm{~s}^{-1}$ for the lower region. The index seems to be harder in the upper region but there is no significant difference when taking into account the errors. In addition to that, we also studied the spectrum of the two specific areas that were defined in H. E. S. S. Collaboration et al. (2016), as shown in Figure 3. The NE and SW quadrants were fitted in two different fits. For each quadrant we subtracted it from the rest of the disk and fitted both the quadrant and the complementary region simultaneously. The analysis of the Fermi-LAT data revealed significant $\gamma$-ray emission at $\sim 4.7 \sigma$ $(T S=22)$ in the NE region but no signal was detected in the $\mathrm{SW}$ region $(T S<3)$. The spectrum of the NE signal is wellfitted by a power law function with a hard index of $1.33 \pm 0.20$ and an energy flux of $\left(1.2 \pm 0.5_{\text {stat }}\right) \times 10^{-11} \mathrm{erg} \mathrm{cm}^{-2} \mathrm{~s}^{-1}$ and we derived a $95 \%$ C.L. upper limit for the SW region $\left(1.09 \times 10^{-12} \mathrm{erg} \mathrm{cm}^{-2} \mathrm{~s}^{-1}\right)$.

\section{RADIO CONTINUUM DATA}

RCW 86 is included in the second Molonglo Galactic Plane Survey (called hereafter MGPS-2) performed by the MOST, at $843 \mathrm{MHz}$ with a bandwidth of $3 \mathrm{MHz}$ and a resolution of $\approx 45 \times 51 \operatorname{arcsec}^{2}$ (Murphy et al. 2007). However, the MOST data are missing structures on scales larger than $\sim 20-30$ arcmin, and this survey does not recover the total radio emission from RCW 86 (its integrated flux density is $\approx 20 \mathrm{Jy}$ in MGPS-2,whereas $\approx 55 \mathrm{Jy}$ is expected from previous observations, e.g., Caswell et al. 1975). Instead, to obtain radio flux densities for regions of the SNR, we used Parkes survey observations at $2.4 \mathrm{GHz}$, with a resolution of $10.2 \times 10.6 \operatorname{arcmin}^{2}$, from Duncan et al. (1995). The integrated flux density of RCW 86 in this survey is $\approx 25 \mathrm{Jy}$, in reasonable agreement with that expected, showing that this single-dish survey is not missing flux from this source. Given the low resolution of this survey, the flux densities in the NE and SW quadrants of H. E. S. S. Collaboration et al. (2016) were obtained by integrating out to somewhat larger radii of 40 arcmin, which gives $4.3 \mathrm{Jy}$ in the NE quadrant and $10.4 \mathrm{Jy}$ in the SW quadrant.

\section{X-RAY OBSERVATIONS}

To estimate the non-thermal X-ray emission from the NE and $\mathrm{SW}$ regions of RCW 86, we analyzed the spectra of these two regions using data of the EPIC-MOS2 instrument of XMMNewton. Since the SNR is larger than the XMM-Newton field of view the $\mathrm{NE}$ and $\mathrm{SW}$ regions were split over several observations, and we took care of this by using spectral analysis of mutually exclusive regions, which together overlapped entirely with the regions indicated in Figure 3. For the NE we used observations number 0208000101 (2004 January 26th, 59.992 ks) and 0504810301 (2007 August 25th, $72.762 \mathrm{ks})$. For the SW region we used observations 0110010701 (2000 August 16th, 23.314 ks) and 0504810401 (2007 August 23rd, $116.782 \mathrm{ks}$ ). The spectra presented in Figure 5 were extracted using the standard XMMNewton analysis package XMM SAS, version $14.0 .^{55}$ The background spectra were obtained from empty regions in the field, taken from the same observations. The extracted spectra

\footnotetext{
55 See http://xmm.esac.esa.int/sas/ for more information about the software
} 

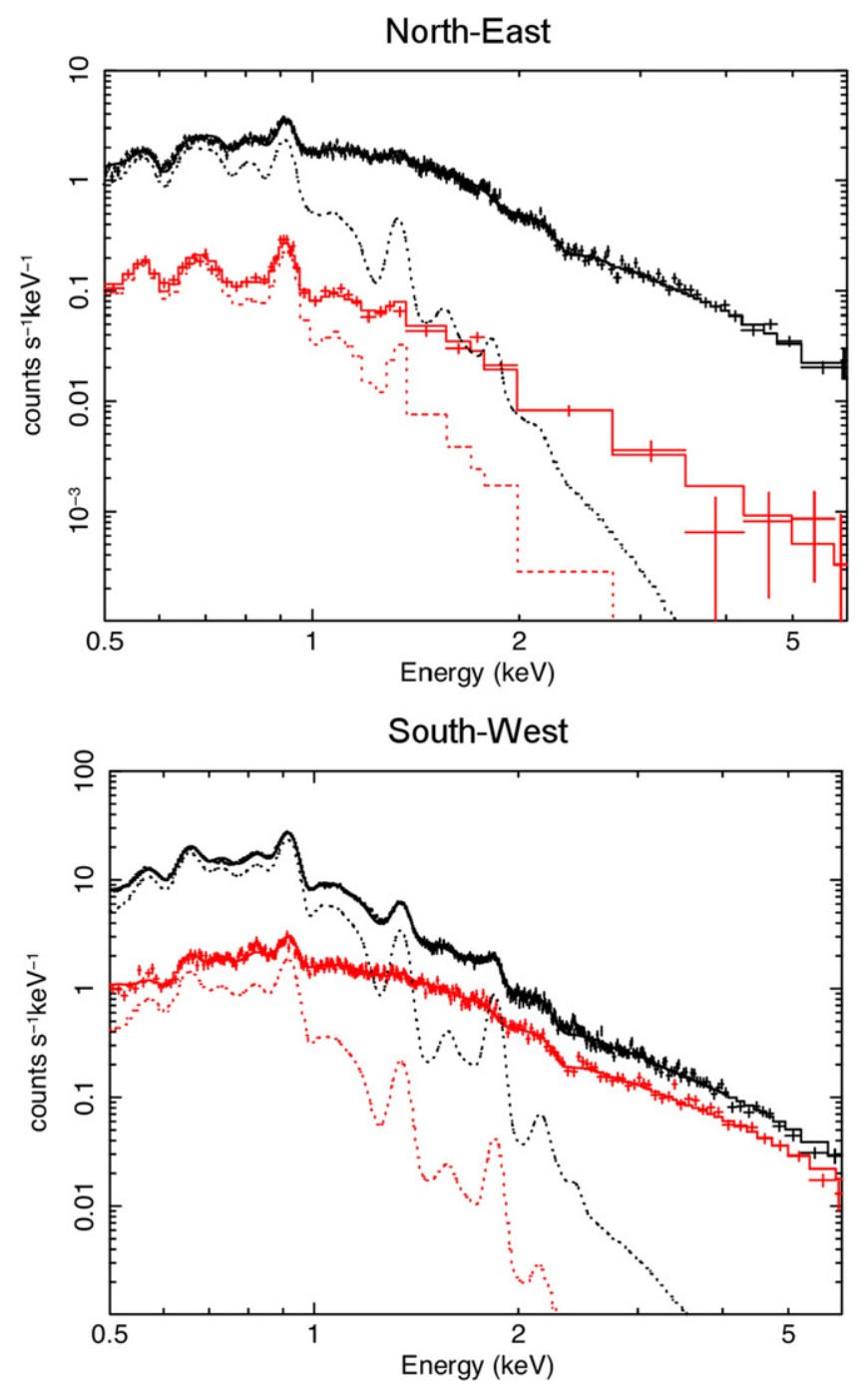

Figure 5. Observed spectra for the split northern regions (Top) and the southern regions (Bottom). Due to the limited field of view of XMM-Newton, two pointings were needed to cover each quadrant shown in Figure 3, hence the black and red curves in both plots. Solid lines show the spectral fits while the dotted lines give the contributions of the thermal components, which are negligible above $2 \mathrm{keV}$.

were then analyzed with the spectral analysis software xspec 12.8 (Arnaud 1996) using the "vnei" model plus power law component, both corrected for Galactic absorption. The absorption columns are $(2.5 \pm 0.1) \times 10^{21} \mathrm{~cm}^{-2}$ for the southern part and $(4.8 \pm 0.1) \times 10^{21} \mathrm{~cm}^{-2}$ for the northern part. From the best fit models we obtained the fluxes in the $3-5 \mathrm{keV}$ band, which for RCW 86 is totally dominated by SC emission. The fluxes are estimated to be $10.0 \times 10^{-12} \mathrm{erg} \mathrm{cm}^{-2} \mathrm{~s}^{-1}$ and $4.3 \times 10^{-12} \mathrm{erg} \mathrm{cm}^{-2} \mathrm{~s}^{-1}$ for the $\mathrm{SW}$ and $\mathrm{NE}$ regions, respectively. The flux measurements have errors of the order of $5 \%-10 \%$, mostly dominated by systematic errors, as absolute flux calibration of X-ray instruments is accurate at the $5 \%$ level. For both regions, the power law index is measured to be $3.0 \pm 0.2$, with the error mostly due to variations within each region.

\section{THE SURROUNDING INTERSTELLAR MEDIUM}

We have analyzed the cold neutral gas in the environs of RCW 86 to investigate the characteristics of the surrounding gas. To carry out this search we used data at $\lambda=21 \mathrm{~cm}$ acquired with the Australia Telescope Compact Array (ATCA) on 2002 March 24. To recover the missing short spatial frequencies, the ATCA data were combined in the $u-v$ plane with single dish observations performed with the Parkes radio telescope. The final data are arranged in a cube with an angular resolution of $2.7 \times 2.5$ (R.A. $\times$ decl.) and a $1 \sigma$ rms noise in line-free channels of about $1 \mathrm{~K}$. The cube covers the velocity range -120.00 to $+126.00 \mathrm{~km} \mathrm{~s}^{-1}$ with a velocity resolution of $0.82 \mathrm{~km} \mathrm{~s}^{-1}$.

The whole $\mathrm{H}_{\text {I }}$ cube was inspected searching for imprints in the surrounding medium that might have been produced by the $\mathrm{SN}$ explosion and/or its precursor star. Different morphological signatures can be left in the interstellar gas by these expanding events, like cavities blown up by the stellar wind of the pre-SN, bubbles surrounded by a higher density neutral shell, accelerated clouds seen in projection against the center of the SNR, etc. These kind of features have been identified in association with several Galactic SNRs (see e.g., Park et al. (2013) and references therein). In the case of RCW 86 we detected the presence of an elongated cavity, about 1.5 in size, that runs almost parallel to the Galactic plane, in the velocity interval between $\sim-38 \mathrm{~km} \mathrm{~s}^{-1}$ and $\sim-32 \mathrm{~km} \mathrm{~s}^{-1}$ (all velocities are referred to the Local Standard of Rest, LSR). Within this velocity range, more precisely between $\sim-35 \mathrm{~km} \mathrm{~s}^{-1}$ and $\sim-33 \mathrm{~km} \mathrm{~s}^{-1}$ the SNR appears surrounded by a tenuous, approximately circular $\mathrm{H}_{\mathrm{I}}$ shell with variable brightness distribution. These morphological findings are in very good agreement with the predictions made on the basis of radio continuum, X-rays, infrared observations and hydrodynamic simulations (Vink et al. 1997; Dickel et al. 2001; Williams et al. 2011; Broersen et al. 2014). After applying a circular rotation model for our Galaxy for $l=315^{\circ} .4, b=-2.3$, the LSR radial velocity interval of the observed features translates into a distance of $\sim 2.5 \pm 0.3 \mathrm{kpc}$. This distance is in very good agreement with that previously obtained for RCW 86 on the basis of optical measurements of proper motions of the filaments (Rosado et al. 1996; Sollerman et al. 2003), suggesting that this gas is placed at the same distance as RCW 86. In addition to the morphological signatures, an independent test of the adopted central radial velocity can be done by comparing the absorbing column density $N_{\mathrm{H}}$ integrated between us and the SNR with Yamaguchi's (2011) best fits derived from X-ray observations. From our $\mathrm{H}_{\mathrm{I}}$ data we obtain $N_{\mathrm{H}}=2.6$ $\times 10^{21} \mathrm{~cm}^{-2}$ (for the whole annulus shown in Figure 8), in good concordance with the values $N_{\mathrm{H}}=2.9$ or $2.8 \pm 0.3$ $\times 10^{21} \mathrm{~cm}^{-2}$, obtained from X-ray data (where the different values depend on the model) and the absorption column given in Section 5 for the southern region. The apparent discrepancy with the absorption columns mentioned in Section 5 for the northern region can be due to the presence of $\mathrm{H} 2$, a contribution to which $\lambda 21 \mathrm{~cm}$ observations are not sensitive. As the distribution of the molecular gas ( $\mathrm{CO}$ in particular) perpendicular to the plane has a semi-scale of $\sim 55 \mathrm{pc}$ in the inner Galaxy, the presence of some molecular gas fragmented in small isolated clouds is natural at the height of RCW 86 $(\sim 100 \mathrm{pc})$. The line of sight where the X-ray absorption was calculated might have crossed one of these cloudlets. Overall, the fact that these $\mathrm{H}_{\mathrm{I}}$ features simultaneously fulfill morphological and kinematical criteria, strongly suggest that the neutral gas observed in this velocity interval is physically associated with the SNR. 

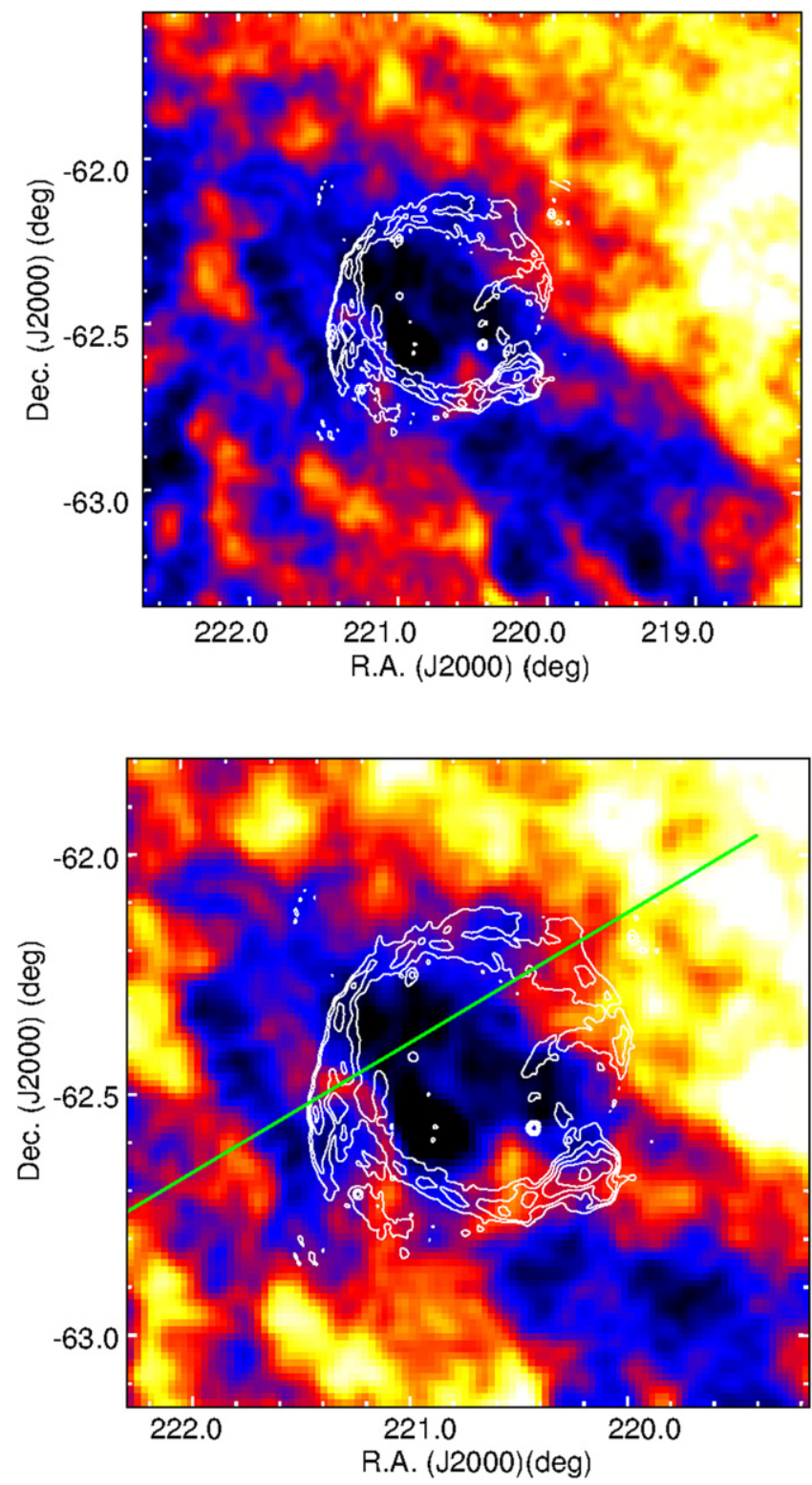

Figure 6. Neutral hydrogen distribution around RCW 86. Top: the HI distribution is displayed with a linear scale between 32.8 and $89.8 \mathrm{~K}$ in an ample field around RCW 86 at the LSR radial velocity of $-34 \mathrm{~km} \mathrm{~s}^{-1}$. Bottom: the same, but in a smaller field in the vicinity of RCW 86, displayed between 32.8 and $73.3 \mathrm{~K}$ to emphasize the presence of the internal shell. The white contours show the radio continuum emission at $843 \mathrm{MHz}$ from the MGPS-2 data. The line included in the bottom panel indicates the direction where the distribution profile shown in Figure 7 was extracted.

Figure 6 (Top) shows the local $\mathrm{H}$ i distribution in a large field (over 5 square degrees) in direction to RCW 86, as observed around the radial velocity of $\sim-34 \mathrm{~km} \mathrm{~s}^{-1}$. The white contours show the radio continuum emission at $843 \mathrm{MHz}$ from the MGPS-2 data. Figure 6 (Bottom) shows the same as in Figure 6 (Top) but in a smaller region around $\mathrm{RCW} 86$ and using a different scale so as to emphasize the fainter inner shell. Figure 7 displays a radial profile traced across the line shown in Figure 6 (Bottom). The arrows indicate the approximate locations of the walls of the outer cavity and the inner shell.

The H I observations can be used to carry out independent estimates of the volume density of the SNR environs. We considered four regions corresponding to the four quadrants of

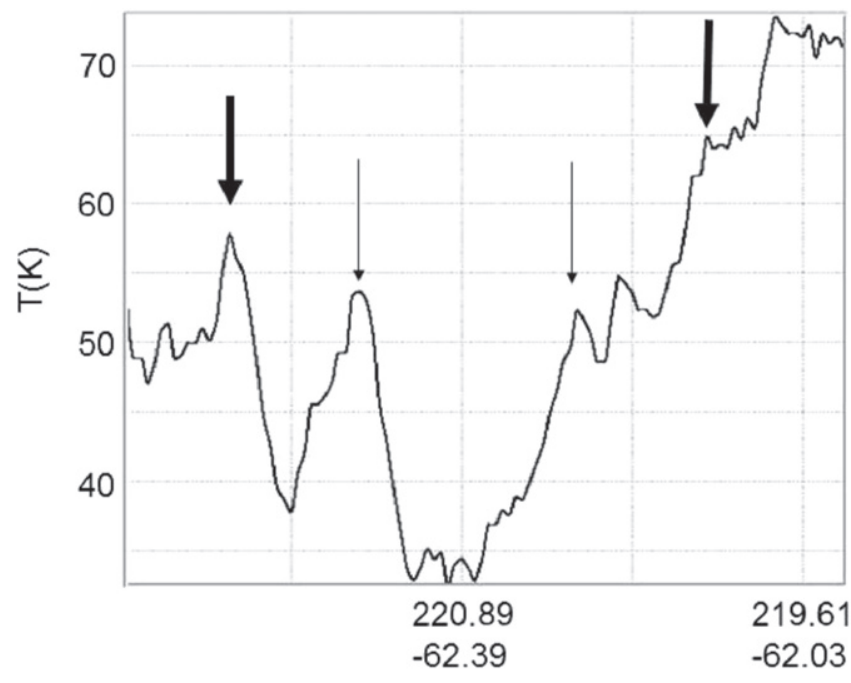

R.A, Dec (J2000) (deg)

Figure 7. Radial profile of the HI distribution measured along the green line showed in Figure 6 (Right). The arrows indicate the presence of an inner shell within a more extended cavity. The $x$-axis corresponds to the equatorial coordinates (in degrees) while the $y$-axis shows the temperature (in kelvin).

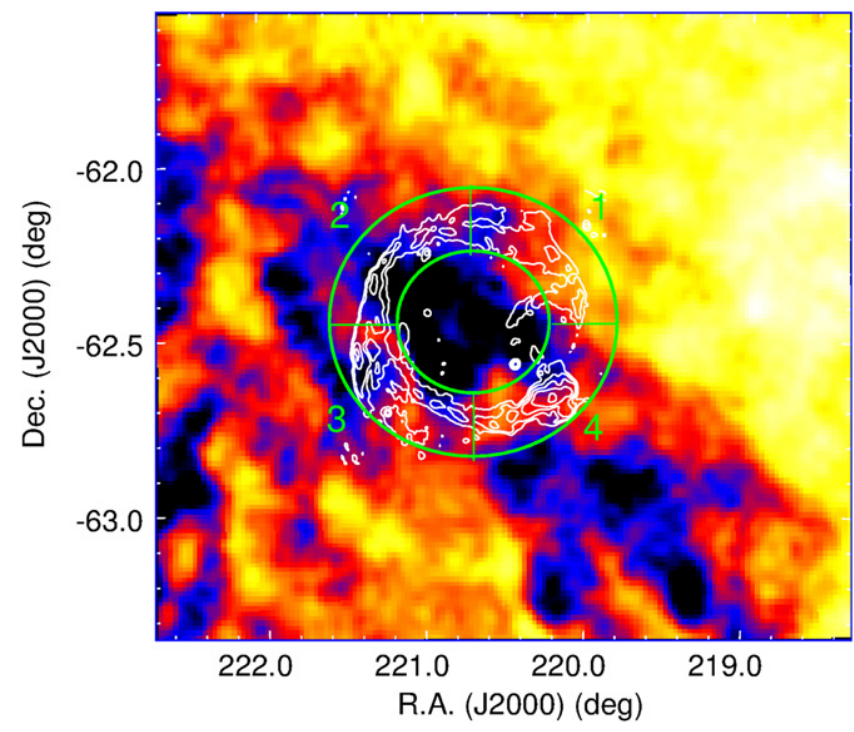

Figure 8. Neutral hydrogen distribution around RCW 86 at the LSR radial velocity of $-34 \mathrm{~km} \mathrm{~s}^{-1}$. The green annulus delimits the regions where the atomic density was estimated.

two concentric circles traced with inner and outer radii coincident with the radio shell (as shown in Figure 8). In this estimate, two aspects have to be considered: the background emission contribution, that takes into account emission that may come from far gas whose emission is detected at the same radial velocities in this direction of the Galaxy, and the geometry of the associated gas along the line of sight. For the first issue we subtracted a uniform background of $T=25 \mathrm{~K}$, a value estimated from the inspection in the observed field of regions free of structures down to the angular resolution of the data. This assumption is reasonable since the neutral gas located at the far distance is at a height well below the Galactic plane in the direction to $b=-2.3$. Concerning the threedimensional distribution of the adjacent gas, we tested the two usually adopted geometries: a cylindrical ring with a depth 
similar to the SNR diameter (the case where the gas accompanies a barrel-shaped SNR), and a spherical shell surrounding the SNR. This last case is consistent with the geometry suggested by the Balmer-dominated filaments that encircle almost the complete periphery of RCW 86 (Smith 1997). It is known that the Balmer-dominated filaments arise from relatively high velocity shocks passing through partially neutral gas. It is natural then to assume that the cold H I mimics the optically depicted SNR. In any case in both considered geometries the results obtained were very similar and in what follows we list an average between the two results.

For region 1 (NW quadrant) $n_{\mathrm{H}} \sim 1.5 \mathrm{~cm}^{-3}$; for region 2 (NE quadrant) $n_{\mathrm{H}} \sim 1 \mathrm{~cm}^{-3}$; for region 3 (SE quadrant) $n_{\mathrm{H}} \sim 1 \mathrm{~cm}^{-3}$ and for region 4 (SW quadrant) $n_{\mathrm{H}} \sim 1.2 \mathrm{~cm}^{-3}$. In all cases the intrinsic error of the quoted numbers is of about $30 \%$ taking into account the uncertainty in the distance (of $25 \%$ ) and the approximate background subtraction. For the interior of the SNR we estimated $n_{\mathrm{H}} \sim 0.5 \mathrm{~cm}^{-3}$.

The complete analysis of the $\mathrm{H}_{\mathrm{I}}$ in the direction of RCW 86 will be published elsewhere.

\section{DISCUSSION}

\subsection{Broadband Modeling}

The main difficulty in determining the origin of the $\gamma$-ray emission of RCW 86 lies in the competition of two major channels of $\gamma$-ray production: the IC scattering of high energy leptons on local photon fields (leptonic scenario) or the decay of neutral pions produced by the interaction between accelerated protons and interstellar clouds located near the remnant (hadronic scenario). We performed a broadband modeling of the non-thermal emission of RCW 86 using radio, X-ray and VHE $\gamma$-ray data, in addition to the FermiLAT observations. This modeling (shown in Figure 9) aims to constrain key parameters such as the average magnetic field and the fraction of the total SN energy which is transferred into protons and electrons. The particle spectra are assumed to follow a power-law function with an exponential cutoff $d N / d E \propto E^{-\Gamma} \times \exp \left(-E / E_{\max }\right)$ with the same index for both distributions (electron and proton), starting at $511 \mathrm{keV}$ for the electrons and $1 \mathrm{GeV}$ for protons. The escape of accelerated particles confined in the magnetic field of the shock is taken into account, assuming a shell thickness of 0.1 . This value was obtained by fitting the Fermi-LAT data with a ring model and is in agreement with H. E. S. S. Collaboration's (2016) estimates. We define $\eta_{\mathrm{e}, \mathrm{n}}$ as the ratio of the total energy injected into accelerated particles $W_{\mathrm{e}, \mathrm{p}}$ to the standard energy of a SN Ia explosion $E_{\mathrm{SN}}$, assumed to be $10^{51}$. The so-called electron-to-proton ratio $K_{\text {ep }}$ is also computed, at momentum $1 \mathrm{GeV} \mathrm{c}{ }^{-1}$ and may be compared to the value measured in CRs $\left(K_{\text {ep }} \sim 10^{-2}\right)$.

\subsection{Modeling of the Whole SNR}

Here we present the results of two leptonic scenario models. Since a pure hadronic scenario requires unlikely parameter values such as a very hard spectral index for protons (as it was already suggested in Lemoine-Goumard et al. 2012) and a high magnetic field $(B>100 \mu \mathrm{G})$, we did not consider this case. The presence of a high magnetic field is not excluded in very thin regions, near the shock, but it is very unlikely to have such high values for the whole remnant. Moreover, a spectral index softer than 1.7 is excluded with more than $3 \sigma$, as described in
Section 3.2. The hadronic model relying on the interactions between escaped protons and a dense interstellar medium in the vicinity of the remnant, as proposed in Gabici et al. (2009), seems also ruled out by the non-detection of molecular clouds in the NE part of the remnant, where the $\gamma$-ray signal detected by the Fermi-LAT is the most important.

Figure 9 shows the result for a one-zone model (top) in which we assumed that SC and IC photons are produced by electrons confined in the same emitting region with a constant magnetic field and a two-zone model (bottom) in which we considered two different populations of radio, X-ray and $\gamma$-ray emitting particles. Parameters of the latter population were obtained with a $\chi^{2}$ fit without considering the radio emitting population for which parameters were determined afterwards in respect to the previous results. The two-zone model is motivated by the bad correlation between the radio and the $\gamma$ ray data, as shown in Section 3, and by several publications which reported large variations of the physical conditions in RCW 86 (Vink et al. 2006; Broersen et al. 2014). To be more conservative on the fraction of the energy injected in protons, the only photon field that was taken into account for the IC scattering of electrons is the Cosmic Microwave Background (CMB). The best-fit parameters for these two models are given in Table 4. For the whole remnant, the radio points in the one-zone model imply a soft spectral index ( 2.4) which would lead to a very strong bremsstrahlung component below $1 \mathrm{GeV}$. To reconcile this low energy component with the new Fermi-LAT upper limit at $1 \mathrm{GeV}$ derived in Section 3.2, a maximum density of $0.1 \mathrm{~cm}^{-3}$ needs to be assumed. In the case of a two-zone model, we obtained a more reasonable index $(\sim 2.2)$ with a density of $1.0 \mathrm{~cm}^{-3}$ and an energy of $2 \%$ of $E_{\mathrm{SN}}$ that goes into protons. Considering a distance of $2.5 \mathrm{kpc}$ and a shell thickness of $0{ }^{\circ}$, the energy density of CRs is estimated at $\sim 40 \mathrm{eV} \mathrm{cm}^{-3}$. In both cases, the magnetic field is around $10 \mu \mathrm{G}$, in agreement with previous modeling by Lemoine-Goumard et al. (2012), Yuan et al. (2014) and H. E. S. S. Collaboration et al. (2016).

\subsection{Modeling of the NE and SW Regions}

In addition to the modeling of the whole SNR, we studied the broadband signal emitted by the NE and SW regions defined in H. E. S. S. Collaboration et al. (2016) and for which we performed a spectral analysis in Section 3.2 of this paper. In this work, we gathered radio (Section 4), X-ray (Section 5), $\mathrm{GeV}$ (Section 3.2) and $\mathrm{TeV}$ data (H. E. S. S. Collaboration et al. 2016) for these two regions and performed a modeling assuming that each region sees a different population of emitting particles. The spectral points for the NE region were obtained by dividing the $100 \mathrm{MeV}-500 \mathrm{GeV}$ energy range into three logarithmically spaced bins only (instead of nine for the whole remnant) because of the reduced statistics. We derived a 95\% C.L. upper limit for the first bin and obtained significant fluxes for the two other bins. To limit the number of fitted parameters, the density was assumed to be of $1.0 \mathrm{~cm}^{-3}$ for both regions (which is in agreement with the values derived in Section 6). We decided to use the best index previously obtained for the whole remnant, in the case of a two-zone model, (2.21) and fixed it for both electron and proton distributions for the two regions, since there is no evidence in favor of different injection slopes in the remnant. The energy injected in protons was fixed to $0.5 \%$ of $E_{\mathrm{SN}}$ (which corresponds to a quarter of the value used for the whole 

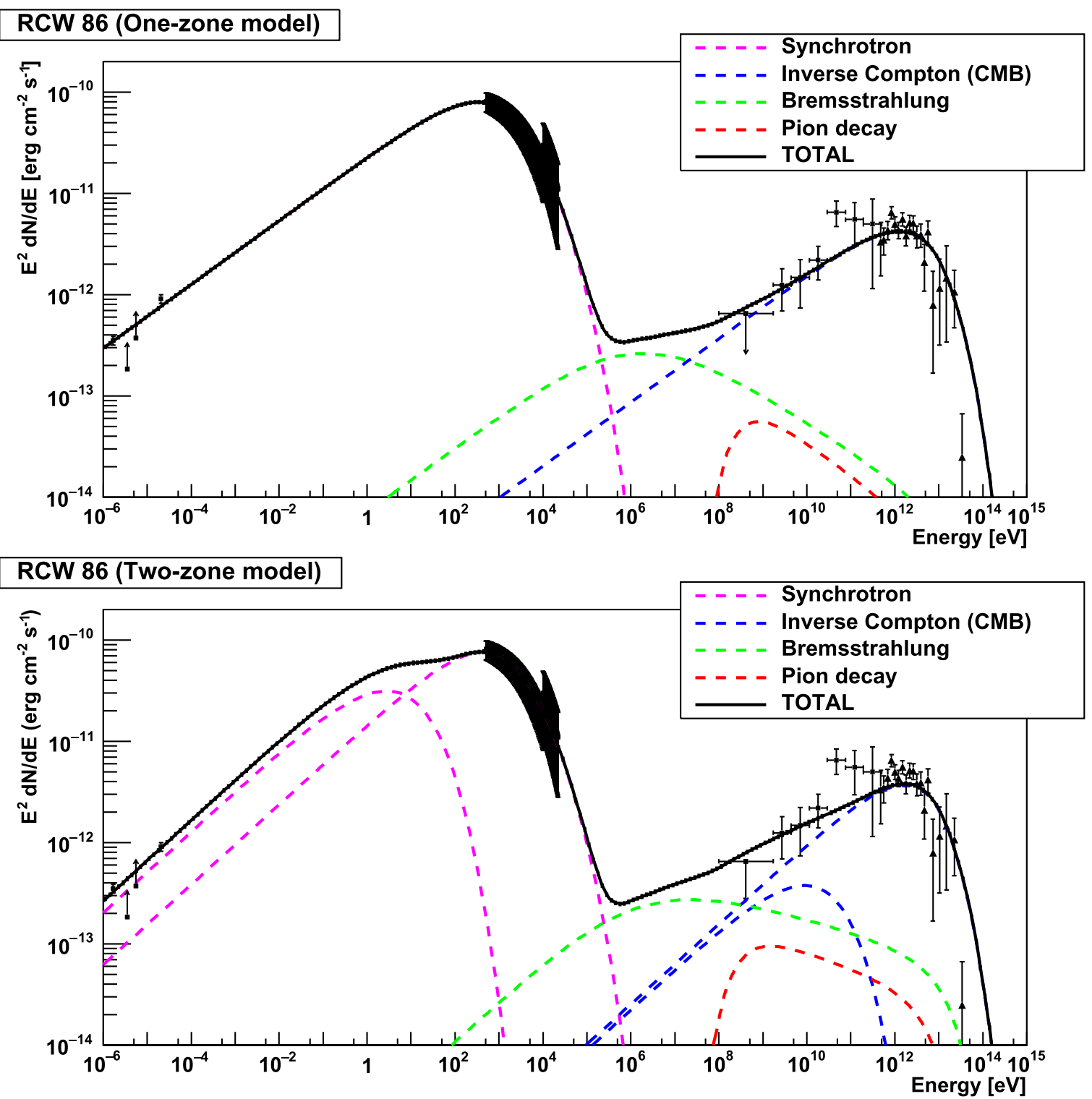

Figure 9. Spectral Energy Distribution of RCW 86 with the best-fit leptonic one-zone (top) and two-zone (bottom) models. The radio points are from Molonglo at $408 \mathrm{MHz}$ and Parkes at $5 \mathrm{GHz}$ (Caswell et al. 1975; Lemoine-Goumard et al. 2012) and lower limits from MOST at $843 \mathrm{MHz}$ and ATCA at $1.43 \mathrm{GHz}$ (Whiteoak \& Green 1996; Dickel et al. 2001). X-ray spectra from ASCA and RXTE are from Lemoine-Goumard et al. (2012). The Fermi-LAT spectral points and upper limits (95\% C.L.) derived in Section 3.2 and the H.E.S.S. data points in the VHE $\gamma$-ray domain from H. E. S. S. Collaboration et al. (2016) are also shown.

Table 4

Values of the Parameters for the Different Modelings of the Broadband Spectrum of RCW 86

\begin{tabular}{|c|c|c|c|c|c|}
\hline \multirow[t]{3}{*}{ Parameters } & \multicolumn{3}{|c|}{ Whole Remnant } & \multicolumn{2}{|c|}{ Regions } \\
\hline & \multirow[t]{2}{*}{ One-zone } & \multicolumn{2}{|c|}{ Two-zone } & \multirow[t]{2}{*}{$\mathrm{NE}$} & \multirow[t]{2}{*}{ SW } \\
\hline & & Radio & X-ray & & \\
\hline Density $\left(\mathrm{cm}^{-3}\right)$ & 0.1 & 1.0 & 1.0 & 1.0 & 1.0 \\
\hline B $(\mu \mathrm{G})$ & $10.2 \pm 0.5$ & 24 & $10.5 \pm 0.7$ & $11.6 \pm 0.7$ & $16.8 \pm 2.1$ \\
\hline$\Gamma_{\mathrm{e}, \mathrm{p}}$ & $2.37 \pm 0.03$ & 2.2 & $2.21 \pm 0.1$ & 2.21 & 2.21 \\
\hline$E_{\max }(\mathrm{TeV})$ & $75 \pm 5$ & 2 & $67 \pm 4$ & $40 \pm 5$ & $61 \pm 5$ \\
\hline$\eta_{\mathrm{e}}\left(\%\right.$ of $\left.E_{\mathrm{SN}}\right)$ & $3.84 \pm 0.5$ & 0.03 & $0.37 \pm 0.02$ & $0.14 \pm 0.04$ & $0.16 \pm 0.04$ \\
\hline$\eta_{\mathrm{p}}\left(\%\right.$ of $\left.E_{\mathrm{SN}}\right)$ & 2 & $\cdots$ & 2 & 0.5 & 0.5 \\
\hline$K_{\text {ep }}\left(\times 10^{-2}\right)$ & $11.1 \pm 1.5$ & $\ldots$ & $13.6 \pm 0.5$ & $5.2 \pm 1.5$ & $5.9 \pm 1.5$ \\
\hline
\end{tabular}

SNR) and the density at $1.0 \mathrm{~cm}^{-3}$. Results are shown in Figure 10 and Table 4 summarizes the parameters for the two models, obtained with a $\chi^{2}$ fit.
We note that the magnetic field is slightly higher in the SW than in the NE, implying a magnetic field gradient in a direction away from the Galactic Plane possibly due to the shock 


\section{RCW 86 - NE region}

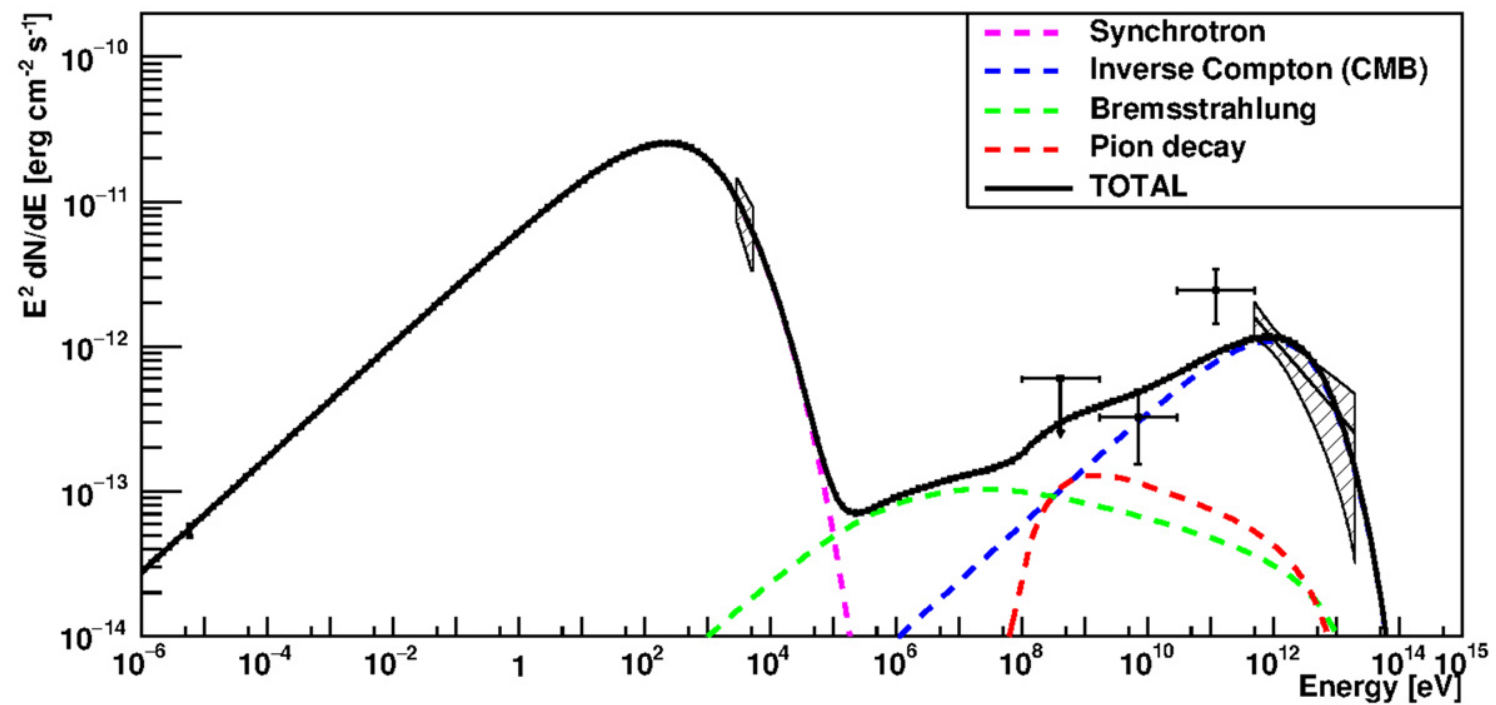

RCW 86 - SW region

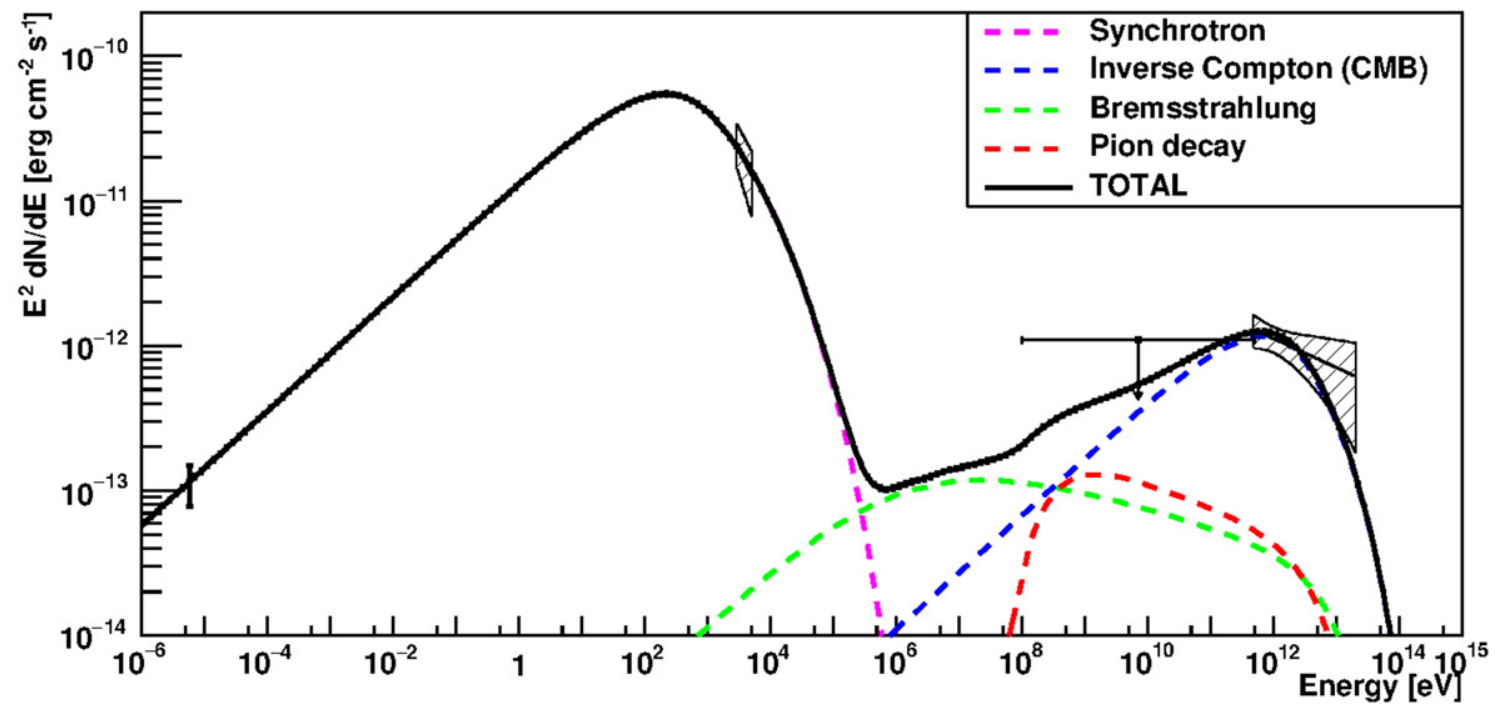

Figure 10. SED modeling for the NE (top) and SW (bottom) regions of RCW 86. The radio fluxes were derived from Parkes observations at $2.4 \mathrm{GHz}$ (as described in Section 4), the X-ray fluxes are estimated using XMM-Newton/MOS data (see Section 5) and the TeV points are from H. E. S. S. Collaboration et al. (2016).

interaction with a denser medium. Another interesting point is that the magnetic field of the NE region is very close to the one obtained for the whole SNR, which is consistent with the fact that most of the GeV emission is detected in the NE part of the remnant. Moreover, $E_{\max }$ is also higher in the $\mathrm{SW}$ than in the $\mathrm{NE}$ which is in agreement with the values of the magnetic field: at early times, when the maximum energy is not limited by SC losses, a higher magnetic field implies a higher $E_{\max }$. Overall, the MWL data indicate variations of the magnetic field within the SNR. The radio emission corresponds to regions with high magnetic fields whereas the $\mathrm{GeV}$ emission detected by FermiLAT corresponds to regions with mixed magnetic fields. And since the H.E.S.S. map shows brighter emission coming from the inside of the remnant than in radio and X-rays, the reverse shock could also be responsible for the CR acceleration but with a lower magnetic field. In the near future, a deep study of RCW 86 with the Cherenkov Telescope Array (Actis et al. 2011) and ASTRO-H (Takahashi et al. 2014) could constrain the magnetic field and provide a precise map of its fluctuation at smaller scales.

\section{CONCLUSIONS}

Analyzing more than 6 years of Fermi-LAT Pass 8 data, we present the first deep study of the morphology and spectrum of the young SNR RCW 86. The spectrum is described by a pure power-law function with an index of $1.42 \pm 0.1_{\text {stat }} \pm 0.06_{\text {syst }}$ in the LAT energy range $(0.1-500 \mathrm{GeV})$. The broadband emission from radio to $\mathrm{TeV}$ cannot be described by a pure hadronic scenario due to the very hard spectral index in the $\mathrm{GeV}$ range, the high magnetic field needed and the lack of a high density medium. The two-zone model provides new constraints on the fraction of the total energy injected in protons and the most conservative value amounts to $\sim 2 \times 10^{49} \mathrm{erg}$ for a density of $1 \mathrm{~cm}^{-3}$. Finally, the nondetection of the SW region of RCW 86 , which is very bright in 
radio, $\mathrm{X}$-rays and at $\mathrm{TeV}$ energies, provides specific constraints on this part of the remnant, in terms of the acceleration mechanism as well as the gas density and the magnetic field.

The Fermi LAT Collaboration acknowledges generous ongoing support from a number of agencies and institutes that have supported both the development and the operation of the LAT as well as scientific data analysis. These include the National Aeronautics and Space Administration and the Department of Energy in the United States, the Commissariat à l'Energie Atomique and the Centre National de la Recherche Scientifique/Institut National de Physique Nucléaire et de Physique des Particules in France, the Agenzia Spaziale Italiana and the Istituto Nazionale di Fisica Nucleare in Italy, the Ministry of Education, Culture, Sports, Science and Technology (MEXT), High Energy Accelerator Research Organization (KEK) and Japan Aerospace Exploration Agency (JAXA) in Japan, and the KA Wallenberg Foundation, the Swedish Research Council and the Swedish National Space Board in Sweden. Additional support for science analysis during the operations phase is gratefully acknowledged from the Istituto Nazionale di Astrofisica in Italy and the Centre National d'Études Spatiales in France. GD and EG are members of CIC-CONICET (Argentina), LD is Fellow of CONICET (Argentina). They are supported through grants from CONICET and ANPCyT (Argentina). We acknowledge to Estela Reynoso and Anne Green who collaborated in the first stages of the HI data acquisition and preocessing.

\section{REFERENCES}

Acero, F., Ackermann, M., Ajello, M., et al. 2015a, ApJS, 218, 23 Acero, F., Ackermann, M., Ajello, M., et al. 2015b, arXiv:1511.06778

Ackermann, M., Ajello, M., Albert, A., et al. 2012, ApJS, 203, 4

Actis, M., Agnetta, G., Aharonian, F., et al. 2011, ExA, 32, 193

Aharonian, F., Akhperjanian, A. G., de Almeida, U. B., et al. 2009, ApJ, 692, 1500

Amato, E., \& Blasi, P. 2006, MNRAS, 371, 1251

Arnaud, K. A. 1996, in ASP Conf. Ser. 101, Astronomical Data Analysis Software and Systems V, ed. G. H. Jacoby, \& J. Barnes (San Francisco, CA: ASP), 17

Atwood, W., Albert, A., Baldini, L., et al. 2013, in 4th Fermi Symp. Proc.eConf C121028 (arXiv:1303.3514)

Atwood, W. B., Abdo, A. A., Ackermann, M., et al. 2009, ApJ, 697, 1071

Axford, W. I., Leer, E., \& Skadron, G. 1977, Proc. ICRC, 11, 132
Ballet, J. 2006, AdSpR, 37, 1902

Bell, A. R. 1978a, MNRAS, 182, 147

Bell, A. R. 1978b, MNRAS, 182, 443

Bell, A. R. 2004, MNRAS, 353, 550

Blandford, R. D., \& Ostriker, J. P. 1978, ApJL, 221, L29

Broersen, S., Chiotellis, A., Vink, J., \& Bamba, A. 2014, MNRAS, 441, 3040

Caprioli, D., Blasi, P., Amato, E., \& Vietri, M. 2008, ApJL, 679, L139

Caswell, J. L., Clark, D. H., Crawford, D. F., \& Green, A. J. 1975, AuJPA, 37,1

Dickel, J. R., Strom, R. G., \& Milne, D. K. 2001, ApJ, 546, 447

Duncan, A. R., Stewart, R. T., Haynes, R. F., \& Jones, K. L. 1995, MNRAS, 277, 36

Gabici, S., Aharonian, F. A., \& Casanova, S. 2009, MNRAS, 396, 1629

Ghavamian, P., Raymond, J., Smith, R. C., \& Hartigan, P. 2001, ApJ, 547, 995

Ginzburg, V. L., \& Syrovatskii, S. I. 1964, AZh, 41, 430

Ginzburg, V. L., \& Syrovatsky, S. I. 1961, PThPS, 20, 1

Helder, E. A., Vink, J., Bamba, A., et al. 2013, MNRAS, 435, 910

Helder, E. A., Vink, J., Bassa, C. G., et al. 2009, Sci, 325, 719

H. E. S. S. Collaboration, Abramowski, A., Aharonian, F., et al. 2016, A\&A, in press (arXiv:1601.04461)

Kerr, M. 2011, PhD thesis, Univ. Washington

Kesteven, M. J., \& Caswell, J. L. 1987, A\&A, 183, 118

Krymskii, G. F. 1977, DoSSR, 234, 1306

Lemoine-Goumard, M., Renaud, M., Vink, J., et al. 2012, A\&A, 545, A28

Long, K. S., \& Blair, W. P. 1990, ApJL, 358, L13

Malkov, M. A., \& Drury, L. O. 2001, RPPh, 64, 429

Mattox, J. R., Bertsch, D. L., Chiang, J., et al. 1996, ApJ, 461, 396

Mills, B. Y., Slee, O. B., \& Hill, E. R. 1961, AuJPh, 14, 497

Murphy, T., Mauch, T., Green, A., et al. 2007, MNRAS, 382, 382

Park, G., Koo, B.-C., Gibson, S. J., et al. 2013, ApJ, 777, 14

Pisarski, R. L., Helfand, D. J., \& Kahn, S. M. 1984, ApJ, 277, 710

Rho, J., Dyer, K. K., Borkowski, K. J., \& Reynolds, S. P. 2002, ApJ, 581,1116

Rodgers, A. W., Campbell, C. T., \& Whiteoak, J. B. 1960, MNRAS, 121, 103

Rosado, M., Ambrocio-Cruz, P., Le Coarer, E., \& Marcelin, M. 1996, A\&A, 315,243

Smith, R. C. 1997, AJ, 114, 2664

Sollerman, J., Ghavamian, P., Lundqvist, P., \& Smith, R. C. 2003, A\&A, 407, 249

Stephenson, F. R., \& Green, D. A. 2002, Historical Supernovae and Their Remnants, Vol. 5 (Oxford: Clarendon)

Takahashi, T., Mitsuda, K., Kelley, R., et al. 2014, Proc. SPIE, 9144, 25

The Fermi-LAT Collaboration 2015, ApJ, submitted (arXiv:1508.04449)

Ueno, M., Sato, R., Kataoka, J., et al. 2007, PASJ, 59, 171

Vink, J. 2012, A\&ARv, 20, 49

Vink, J., Bleeker, J., van der Heyden, K., et al. 2006, ApJL, 648, L33

Vink, J., Kaastra, J. S., \& Bleeker, J. A. M. 1997, A\&A, 328, 628

Whiteoak, J. B. Z., \& Green, A. J. 1996, A\&AS, 118, 329

Williams, B. J., Blair, W. P., Blondin, J. M., et al. 2011, ApJ, 741, 96

Yamaguchi, H., Koyama, K., Nakajima, H., et al. 2008, PASJ, 60, 123

Yamaguchi, H., Koyama, K., \& Uchida, H. 2011, PASJ, 63, 837

Yuan, Q., Huang, X., Liu, S., \& Zhang, B. 2014, ApJL, 785, L22 